General Mathematics Vol. 27, No. 2 (2019), 13-21

DOI:10.2478/gm-2019-0011

sciendo

\title{
Certain basic information related to the Tremblay operator and some applications in connection therewith ${ }^{1}$
}

\author{
Hüseyin Irmak
}

\begin{abstract}
In this scientific note, an operator, which is the well-known Tremblay operator in the literature, is first introduced and some of its applications to certain analytic complex functions, which are normalized and analytic in the open unit disk, are then determined. In addition, certain special results of the related applications are also emphasized.
\end{abstract}

2010 Mathematics Subject Classification: 26A33, 34A08, 35G10, 35F05, 30C55, 30C45, 30A10.

Key words and phrases: Complex plane, Domain in the complex plane, Normalized analytic function, Fractional calculus, the Tremblay operator, Equations and inequalities in the complex plane.

\section{Introduction and motivation}

As it is known from literature, the well-known fractional calculus (FC) is a generalization of the classical calculus concerned with operations of integration and also differentiation of integer or non-integer (fractional) order. The concept of fractional operators, which are constituted by FC, has been introduced almost simultaneously with the development of the classical ones. The first known reference can be found in the correspondence of G. W. Leibniz and Marquis de L'Hospital in 1695, where

\footnotetext{
${ }^{1} 18$ May, 2019

Accepted for publication (in revised form) 16 December, 2019
} 
the question of meaning of the semi-derivative has been raised. This question consequently attracted the interest of many well-known mathematicians, including Euler, Liouville, Laplace, Riemann, Grünwald, Letnikov and many others. Since the 19th century, the theory of FC developed rapidly, mostly as a foundation for a number of applied disciplines, including fractional geometry, fractional differential equations (FDE), i.e., differential equations determined by FC, and fractional dynamics.

In the literature, several works consisting of theoretical, practical results and, specially new definitions, which are determined by FC ( or FDE), can be encountered, and, besides, there are several results consisting of some relations between certain novel results constituted by fractional or ordinary differential operators. In this note, as a novel example or certain applications of FC (or FDE), one of those is first reminded and some of its applications are also presented. For this, there is a need to recall or to introduce some information and also definitions regarding to certain applications of, specially, the Trembley operator constituted by FC. For certain operators and their applications, one can check certain works in [2], [5] and [7]-[9].

For this investigation relating to the Tremblay operator, there is a need to recall certain basic notations and well-known definitions.

\section{Notations, definitions and lemmas}

First of them, there is a need to introduce some notations and definitions. For those, let $\mathbb{C}, \mathbb{R}, \mathbb{N}$ and $\mathbb{U}$ be the set of complex numbers, be the set of real numbers, be the set of positive integers and be the open unit disk, i.e., the open set in:

$$
\mathbb{U}:=\{z: z \in \mathbb{C} \text { and }|z|<1\}
$$

respectively.

Moreover, let $\mathcal{A}_{n}$ denote the family of the (complex valued) functions $f(z)$ normalized by the following Taylor-Maclaurin series:

$$
f(z)=z+a_{n+1} z^{n+1}+a_{n+2} z^{n+2}+\cdots \quad\left(a_{n+1} \in \mathbb{C} ; n \in \mathbb{N}\right),
$$

which are analytic (and univalent) in the domain $\mathbb{U}$ and also let $\mathcal{H}_{q}$ denote the family of (complex valued) functions $g(z)$ in the following Taylor-Maclaurin:

$$
g(z)=q+b_{n} z^{n}+b_{n+1} z^{n+1}+\cdots \quad\left(q \in \mathbb{C} ; b_{n} \in \mathbb{C} ; n \in \mathbb{N}\right),
$$

which are analytic (and univalent) in the the domain $\mathbb{U}$.

We next recall, for a complex function $\kappa:=\kappa(z)$, in general, the definition of fractional derivative is denoted by the symbol:

$$
\mathcal{D}_{z}^{\mu}[\kappa] \quad(0 \leq \mu<1)
$$

and also defined as follows (cf., e.g., [14], [15], [1]-[3], [7]-[12]): 
Let the function $\kappa(z)$ be analytic in a simply-connected region of the $z$-plane containing the origin. Then, the fractional derivative of order $\mu$ is defined by

$$
\mathcal{D}_{z}^{\mu}[\kappa]=\frac{1}{\Gamma(1-\mu)} \frac{d}{d z} \int_{0}^{z} \frac{\kappa(\xi)}{(z-\xi)^{\mu}} d \xi \quad(0 \leq \mu<1),
$$

where the multiplicity of $(z-\xi)^{-\mu}$ above is removed requiring $\log (z-\xi)$ to be real when $z-\xi>0$. All right, here and throughout this paper, the function $\Gamma$ is the well-known gamma function in the literature.

Under favour of the definition given in (1), for an analytic function $\kappa(z)$, the well-known derivative, which is the Srivastava-Owa derivative of order $m+\mu$ is then presented by

$$
\mathcal{D}_{z}^{m+\mu}[\kappa]=\frac{d^{m}}{d z^{m}}\left(\mathcal{D}_{z}^{\mu}[\kappa]\right) \quad(0 \leq \mu<1 ; m \in \mathbb{N} \cup\{0\}),
$$

which readily yields

$$
\mathcal{D}_{z}^{0+\mu}[\kappa]=\mathcal{D}_{z}^{\mu}[\kappa] \quad(0 \leq \mu<1)
$$

and

$$
\mathcal{D}_{z}^{1+\mu}[\kappa]=\frac{d}{d z}\left(\mathcal{D}_{z}^{\mu}[\kappa]\right) \quad(0 \leq \mu<1) .
$$

More recently, by means of the Srivastava-Owa derivative in [12] and the Tremblay operator in [5] and [18], certain operators were introduced and also studied as an certain novel applications of fractional derivative's operator:

$$
\mathcal{T}_{\tau, \mu}[\cdot] \quad(0<\tau \leq 1 ; 0<\mu \leq 1 ; 0 \leq \tau-\mu<1),
$$

which was defined in the domain of the complex plane and whose properties in several spaces were discussed systematically. For both the details of FC and, as example, some novel results proven by certain different methods, one may refer to the scientific works given by [1]-[3], [5], [6]-[13] and see also [14]-[18].

For a function $f(z)$ belonging to the class $\mathcal{A}_{n}$, in general, the Tremblay operator:

$$
\mathcal{T}_{\tau, \mu}[f(z)] \quad \text { or } \quad \mathcal{T}_{\tau, \mu}[f]
$$

is denoted and

$$
\mathcal{T}_{\tau, \mu}[f]:=\frac{\Gamma(\mu)}{\Gamma(\tau)} z^{1-\mu} \mathcal{D}_{z}^{\tau-\mu}\left[z^{\tau-1} f(z)\right],
$$

is also defined, where $0<\tau \leq 1,0<\mu \leq 1,0 \leq \tau-\mu<1$ and $z \in \mathbb{U}$.

As a result of a simple research, it is easily seen that the operator:

$$
\mathcal{D}_{z}^{\tau-\mu}[\cdot] \quad(0<\tau \leq 1 ; 0<\mu \leq 1 ; 0 \leq \tau-\mu<1)
$$

is equivalent to the operator, which is the Srivastava-Owa operator of fractional derivative of order $\tau-\mu$ represented by (1). 
In order to prove the main results in relation with the Tremblay opertor introduced by (2), the following lemmas will be required.

Lemma 1. (see [5]) Let a function $f(z)$ be in the class $\mathcal{A}(n)$. Then,

$$
\mathcal{T}_{\tau, \mu}[f(z)]=\frac{\tau}{\mu} z+\sum_{k=n+1}^{\infty} \frac{\Gamma(k+\tau) \Gamma(\mu)}{\Gamma(k+\mu) \Gamma(\tau)} a_{k} z^{k},
$$

where $0<\tau \leq 1,0<\mu \leq 1,0 \leq \tau-\mu<1$, and $z \in \mathbb{U}$.

Lemma 2. (see [5]) Let a function $f(z)$ be in the class $\mathcal{A}(n)$. Then,

$$
\frac{d}{d z}\left(\mathcal{T}_{\tau, \mu}[f(z)]\right)=\frac{\tau}{\mu}+\sum_{k=n+1}^{\infty} \frac{k \Gamma(k+\tau) \Gamma(\mu)}{\Gamma(k+\mu) \Gamma(\tau)} a_{k} z^{k-1},
$$

where $0<\tau \leq 1,0<\mu \leq 1,0 \leq \tau-\mu<1$, and $z \in \mathbb{U}$.

Lemma 3. (see [15]) Let a function $g(z)$ be in the class $\mathcal{H}:=\mathcal{H}_{1}$. If there exists a point $z_{0}$ in $\mathbb{U}$ such that

$$
\Re e(g(z))>0\left(|z|<\left|z_{0}\right|<1\right) \quad, \quad \Re e\left(g\left(z_{0}\right)\right)=0 \quad \text { and } \quad g\left(z_{0}\right) \neq 0,
$$

then

$$
g\left(z_{0}\right)=i v \quad \text { and }\left.\quad z g^{\prime}(z)\right|_{z=z_{0}}=\left.i \frac{\omega}{2}\left(v+\frac{1}{v}\right) g(z)\right|_{z=z_{0}}
$$

where $\omega \geq 1$ and $v \in \mathbb{R}^{*}:=\mathbb{R}-\{0\}$.

In view of the information above, it is clear that

$$
\frac{\mathcal{T}_{\tau, \mu}[f]}{z} \in \mathcal{H}_{\tau / \mu}
$$

and

$$
\frac{d}{d z}\left(\mathcal{T}_{\tau, \mu}[f]\right) \in \mathcal{H}_{\tau / \mu}
$$

and, specially,

$$
\begin{gathered}
\mathcal{T}_{1,1}[f]=f(z) \in \mathcal{A}_{n}, \\
\frac{d}{d z}\left(\mathcal{T}_{1,1}[f]\right)=f^{\prime}(z) \in \mathcal{H}_{1}
\end{gathered}
$$

and also

$$
\frac{\mathcal{T}_{1,1}[f]}{z}=\frac{f(z)}{z} \in \mathcal{H}_{1},
$$

where $f \equiv f(z) \in \mathcal{A}_{n}, 0<\tau \leq 1,0<\mu \leq 1,0 \leq \tau-\mu<1$, and $z \in \mathbb{U}$. 


\section{Main results and implications}

We now begin by setting and then by proving the main results associating with certain complex equations or inequalities, which are specified by the Tremblay operator, defined as in (2), for functions in the family $\mathcal{A}_{n}$.

Theorem 1. Let $\Phi(z)$ be a function defined as the complex equation given in the form:

$$
z \frac{d^{2}}{d z^{2}}\left(\mathcal{T}_{\tau, \mu}[f]\right)+\frac{d}{d z}\left(\mathcal{T}_{\tau, \mu}[f]\right)-\Phi(z)=0
$$

and also let the following condition:

$$
\Re e(\Phi(z))>\frac{1}{2}\left(3 \kappa-\frac{\tau}{\mu}\right)
$$

satisfy for some $z(z \in \mathbb{U}), \kappa(0 \leq \kappa<1), \mu(0<\mu \leq 1)$ and $\tau(0<\tau \leq 1)$, where $0 \leq \tau-\mu<1$ and $f \in \mathcal{A}_{n}$.

Then, the following inequality:

$$
\Re e\left(\frac{d}{d z}\left(\mathcal{T}_{\tau, \mu}[f]\right)\right)>\kappa \quad\left(0 \leq \kappa<1 ; f \equiv f(z) \in \mathcal{A}_{n} ; z \in \mathbb{U}\right)
$$

is also satisfied.

textbfProof. In the light of (1) and (2), let us define a function $q(z)$ as in the following-implicit form:

$$
\frac{d}{d z}\left(\mathcal{T}_{\tau, \mu}[f]\right)=\kappa+\left(\frac{\tau}{\mu}-\kappa\right) q(z),
$$

where $f \in \mathcal{A}_{n}, z \in \mathbb{U}, 0 \leq \kappa<1,0<\mu \leq 1,0<\tau \leq 1$ and $0 \leq \tau-\mu<1$. When taking into consideration the form of the function $q(z)$, defined in the form (10), it is easily seen that the function $q(z)$ is in the family $\mathcal{H}_{1}$. Namely, it is an analytic function in $\mathbb{U}$ and $q(0)=1$. In the light of (3) of Lemma 1 and also (4) of Lemma 2 , from (10), it follows that

$$
z \frac{d^{2}}{d z^{2}}\left(\mathcal{T}_{\tau, \mu}[f]\right)=\left(\frac{\tau}{\mu}-\kappa\right) z \frac{d}{d z}(q(z)) .
$$

Thus, the results in (10) and also (11) give us

$$
\begin{aligned}
z \frac{d^{2}}{d z^{2}}\left(\mathcal{T}_{\tau, \mu}[f]\right) & +\frac{d}{d z}\left(\mathcal{T}_{\tau, \mu}[f]\right) \\
= & \left(\frac{\tau}{\mu}-\kappa\right) z \frac{d}{d z}(q(z))+\kappa+\left(\frac{\tau}{\mu}-\kappa\right) q(z)
\end{aligned}
$$




$$
=: \Phi(z) \quad \text { (say.) }
$$

Suppose now that there exists a point $z_{0}$ satisfying the condition:

$$
\Re e\left(z_{0}\right)=0 \quad\left(z_{0} \in \mathbb{U}\right),
$$

given by (5) of Lemma 3. Then, by applying the assertions given by (6) of Lemma 3 , which are

$$
\begin{gathered}
q\left(z_{0}\right)=i v \quad \text { and }\left.\quad z q^{\prime}(z)\right|_{z=z_{0}}=\left.i \omega\left(v+\frac{1}{v}\right) q(z)\right|_{z=z_{0}} \\
\left(\omega \geq 1 / 2 ; v \in \mathbb{R}^{*} ; z_{0} \in \mathbb{U}\right)
\end{gathered}
$$

in the last equation given by (13), the following results:

$$
\Phi\left(z_{0}\right)=\left(\frac{\tau}{\mu}-\kappa\right) z_{0} q^{\prime}\left(z_{0}\right)+\kappa+\left(\frac{\tau}{\mu}-\kappa\right) q\left(z_{0}\right),
$$

or, equivalently,

$$
\Phi\left(z_{0}\right)=-\omega\left(1+v^{2}\right)\left(\frac{\tau}{\mu}-\kappa\right)+\kappa+\left(\frac{\tau}{\mu}-\kappa\right) i v
$$

are then obtained.

By considering the values of the parameters $\omega$ and $v$, the real part of $\Phi\left(z_{0}\right)$, given by (13), follows that

$$
\begin{aligned}
\Re e\left(\Phi\left(z_{0}\right)\right) & =\kappa-\omega\left(1+v^{2}\right)\left(\frac{\tau}{\mu}-\kappa\right) \\
& \leq \kappa-\frac{1+v^{2}}{2}\left(\frac{\tau}{\mu}-\kappa\right) \\
& \leq \kappa-\frac{1}{2}\left(\frac{\tau}{\mu}-\kappa\right) \\
& =\frac{1}{2}\left(3 \kappa-\frac{\tau}{\mu}\right),
\end{aligned}
$$

which is a contradiction with the hypothesis of Theorem 1, given by (8). Thus, there is no any point $z_{0}$ such that

$$
\Re e\left\{q\left(z_{0}\right)\right\}=0,
$$

where $z_{0} \in \mathbb{U}$. This means that $\Re e\left\{q\left(z_{0}\right)\right\}>0$ for all $z \in \mathbb{U}$. So, the statement, given by (10), immediately requires the inequality:

$$
\begin{gathered}
\Re e\left(\frac{d}{d z}\left(\mathcal{T}_{\tau, \mu}[f]\right)\right)=\Re e\left(\kappa+\left(\frac{\tau}{\mu}-\kappa\right) q(z)\right)>\kappa \\
(0 \leq \kappa<1 ; z \in \mathbb{U}),
\end{gathered}
$$


which is the inequality given by (9). Thereby, the proof of Theorem 1 is completed.

Theorem 2. Let $\Phi(z)$ be a function defined by the following equation:

$$
z \frac{d}{d z}\left(\mathcal{T}_{\tau, \mu}[f]\right)+\left(\mathcal{T}_{\tau, \mu}[f]\right)-z \Phi(z)=0
$$

and also let the inequality:

$$
\Re e(\Phi(z))>\frac{1}{2}\left(5 \kappa-\frac{\tau}{\mu}\right)
$$

satisfy for all for some $z(z \in \mathbb{U}), \kappa(0 \leq \kappa<1), \mu(0<\mu \leq 1)$ and $\tau(0<\tau \leq 1)$, where $0 \leq \tau-\mu<1$ and $f \in \mathcal{A}_{n}$.

Then, the following inequality:

$$
\begin{gathered}
\Re e\left(\bar{z} \mathcal{T}_{\tau, \mu}[f]\right)>\kappa|z|^{2} \\
\left(0 \leq \kappa<1 ; f \equiv f(z) \in \mathcal{A}_{n} ; z \in \mathbb{U}\right)
\end{gathered}
$$

is satisfied.

Proof. First of all, let $f \in \mathcal{A}_{n}, z \in \mathbb{U}, 0 \leq \kappa<1,0<\mu \leq 1,0<\tau \leq 1$ and $0 \leq \tau-\mu<1$. Then, if one defines a function $q(z)$ as in the implicit form:

$$
\frac{\mathcal{T}_{\tau, \mu}[f]}{z}=\kappa+\left(\frac{\tau}{\mu}-\kappa\right) q(z)
$$

and then follows the all steps used in the proof of Theorem 1, it can be easily arrived at the desired proof. Therefore, it is here omitted.

By a simple focusing on the main results (Theorems 1 and 2), it is easy to see that they include many interesting and useful results in relation with FC, FDE and certain equations or inequalities, which will be obtained by the main results above. So, as certain implications of the main results, i.e., theorems 1 and 2, there can determine several special results by choosing the values of the parameters in both theorems. At the same time, a number of specific results can be also obtained by taking extra condition(s) into consideration for all possible results. We want to present only one of them, which relates to geometric properties of analytic (and univalent) functions. For its details and also some novel examples, see the works in [4], [1]-[3] and [6]-[13].

As a consequence of Theorem 1 , by taking $\tau:=1$ and $\mu:=1$ in Theorem 1 , the following special result can be easily constituted.

Corollary 1. Let $0 \leq \kappa<1, f(z) \in \mathcal{A}_{n}$ and $z \in \mathbb{U}$. If the following inequality:

$$
\Re e\left(z f^{\prime \prime}(z)+f^{\prime}(z)\right)>\frac{3 \kappa-1}{2}
$$

is provided, then

$$
\Re e\left(f^{\prime}(z)\right)>\kappa
$$

is also provided. 


\section{Conclusions}

In the first section of this investigation, various basic information in relation with FC and the Tremblay operator were introduced. In the second section, some results specified by the Tremblay operator were then created. In addition, as certain implications of the related results (Theorems 1 and 2), some extra information were presented. As an example, only one of them, namely, Corollary 1 (above) was also revealed.

\section{References}

[1] O. Altıntaş, H. Irmak, H. M. Srivastava, Fractional calculus and certain starlike functions with negative coefficients, Comput. Math. Appl., vol. 30, no. 2, 1995, $9-15$.

[2] M. P. Chen, H. Irmak, H. M. Srivastava, Some families of multivalently analytic functions with negative coefficients, J. Math. Anal. Appl., vol. 214, no. 2, 1997, 674-690.

[3] M. P. Chen, H. Irmak, H. M. Srivastava, A certain subclass of analytic functions involving operators of fractional calculus, Comput. Math. Appl., vol. 35, no. 2, 1998, 83-91.

[4] P. L. Duren, Grundlehren der Mathematischen Wissenchaffen, Springer-Verlag, New York, Berlin, Heidelberg, Tokyo, 1983.

[5] Z. Esa, H. M. Srivastava, A. Kılıçman, R. W. Ibrahim, A novel subclass of analytic $f$ unctions specified by a family of fractional derivatives in the complex domain, Filomat, vol. 31, no. 9, 2017, 2837-2849.

[6] H. Irmak, Certain complex equations and some of their implications in relation with normalized analytic functions, Filomat, vol. 30, no. 12, 2016, 3371-3376.

[7] H. Irmak, Some novel applications in relation with certain equations and inequalities in the complex plane, Math. Commun., vol. 23, no. 1, 2018, 9-14.

[8] H. Irmak, The ordinary differential operator and some of its applications to pvalently analytic functions, Electron. J. Math. Anal. Appl., vol. 4, no. 1, 2016, 205-210.

[9] H. Irmak, The fractional differ-integral operators and some of their applications to certain multivalent functions, J. Fract. Calc. Appl., vol. 8, no. 1, 2017, 99-107.

[10] H. Irmak, R. K. Raina, Some applications of generalized fractional calculus operators to a novel class of analytic functions with negative coefficients, Taiwanese J. Math., vol. 8, no. 3, 2004, 443-452. 
[11] H. Irmak, B. A. Frasin, An application of fractional calculus and its implications relating to certain analytic functions and complex equations, J. Fract. Calc. Appl., vol. 6, no. 2, 2015, 94-100.

[12] H. Irmak, P. Agarwal, Some comprehensive inequalities consisting of MittagLeffler type functions in the complex plane, Math. Model. Nat. Phenom., vol. 12 , no. $3,2017,65-71$.

[13] H. Irmak, P. Agarwal, Comprehensive Inequalities and Equations Specified by the Mittag-Leffler Functions and Fractional Calculus in the Complex Plane. In: Agarwal P., Dragomir S., Jleli M., Samet B. (eds) Advances in Mathematical Inequalities and Applications. Trends in Mathematics. Birkhauser, Singapore, 2018.

[14] M. Şan, H. Irmak, A note on some relations between certain inequalities and normalized analytic functions, Acta Univ. Sapientiae Math., vol. 10, no. 2, 2018, 368-374.

[15] M. Nunokawa, On properties of non-Caratheodory functions, Proc. Japan Acad. Ser. A Math. Sci., vol. 68, no. 6, 1992, 152-153.

[16] S. Owa, On the distortion theorems. I, Kyungpook Math. J., vol. 8, no. 1, 1978, 53-59.

[17] H. M. Srivastava, S. Owa (Editors), Univalent functions, fractional calculus and their applications, Halsted Press, John Wiley and Sons., New york, Chieschester, Brisbane, Toronto, 1989.

[18] R. W. Ibrahim, J. M. Jahangiri, Boundary fractional differential equation in a complex domain, Boundary Value Prob., Article ID 66, 2014, 1-11.

\section{Hüseyin Irmak}

Çankırı Karatekin University

Faculty of Science

Department of Mathematics

Tr-18100, Uluyazı Campus, Çankırı, TURKEY

e-mail: hirmak@karatekin, hisimya@yahoo.com 